\title{
Recomendaciones de expertos para el tratamiento biológico en pacientes con psoriasis
}

\author{
Nancy Podoswa-Ozerkovsky, ${ }^{1 *}$ Mario Amaya-Guerra, ${ }^{2}$ José F. Barba-Gómez, ${ }^{3}$ Lorena Estrada-Aguilar, ${ }^{4}$ \\ Minerva Gómez-Flores, ${ }^{5}$ Adriana L. Lopeztello-Santillán, ${ }^{6}$ César A. Maldonado-García, ${ }^{7}$ \\ Mónica I. Rivera-Gómez, ${ }^{8}$ Delfina G. Villanueva-Quintero ${ }^{9}$ y Gladys León-Dorantes ${ }^{10}$ \\ ${ }^{1}$ Instituto Mexicano del Seguro Social, Hospital General Regional 1, Servicio de Dermatología, Ciudad de México; ${ }^{2}$ Universidad de Monterrey, \\ Centro de Salud y Desarrollo, Servicio de Dermatología, Nuevo León; ${ }^{3}$ nstituto Dermatológico de Jalisco Dr. José Barba Rubio, Jalisco; ${ }^{4}$ nstituto \\ de Seguridad y Servicios Sociales de los Trabajadores del Estado, Hospital Regional "Lic. Adolfo López Mateos", Servicio de Dermatología, Ciudad \\ de México; ${ }^{5}$ Universidad Autónoma de Nuevo León, Hospital Universitario, Servicio de Dermatología, Nuevo León; ${ }^{6}$ Instituto de Seguridad Social \\ del Estado de México y Municipios, Servicio de Dermatología, Estado de México; ${ }^{7}$ Centro Dermatológico "Dr. Ladislao de la Pascua", Jefatura de \\ la Clínica de la Psoriasis, Ciudad de México; ${ }^{8}$ Instituto Mexicano del Seguro Social, Centro Médico Nacional La Raza, Hospital de Especialidades, \\ Ciudad de México; ${ }^{\circ}$ Centro de Atención en Enfermedades Inflamatorias, Investigación Clínica, Jalisco; ${ }^{10}$ Secretaría de Salud, Unidad de Innovación \\ Clínica y Epidemiológica del Estado de Guerrero, Guerrero. México
}

\section{Resumen}

En los últimos años, la introducción de diversos medicamentos biológicos para el tratamiento de la psoriasis ha aumentado considerablemente el arsenal terapéutico del médico, con lo cual se ha logrado un fuerte impacto positivo en el control de la enfermedad. Con el fin de proveer de las mejores recomendaciones para el uso de estos biológicos en los pacientes afectados de psoriasis, el grupo mexicano de expertos en psoriasis PSOMEX ha formulado recomendaciones para mejorar la comprensión y el posicionamiento terapéutico de este tipo de medicamentos.

PALABRAS CLAVE: Psoriasis. Artritis psoriásica. Recomendaciones. Agentes biológicos. México.

\section{Expert recommendations for biological treatment in patients with psoriasis}

\begin{abstract}
In recent years, the introduction of a series of biological drugs for the treatment of psoriasis has considerably increased the therapeutic armamentarium of doctors, and thus a strongly positive impact on the control of this condition has been achieved. With the purpose to provide the best recommendations for the use of these biological agents in patients with psoriasis, the Mexican group of psoriasis experts, PSOMEX, has developed recommendations in order to improve the understanding and therapeutic positioning of this type of medications.
\end{abstract}

KEY WORDS: Psoriasis. Psoriatic arthritis. Recommendations. Biological agents. Mexico.

\section{Introducción}

La psoriasis es una enfermedad cutánea crónica, con una base inflamatoria inmunológica mediada muy compleja. Es una entidad frecuente, con tasas de prevalencia estimadas de entre 1 y $4 \%$ en la población mundial y está asociada a múltiples comorbilidades, deterioro significativo en la calidad de vida, de la función social y económica, así como a la reducción en la expectativa de vida. ${ }^{1}$

\footnotetext{
Correspondencia:

Fecha de recepción: 30-04-2020

*Nancy Podoswa-Ozerkovsky

Fecha de aceptación: 12-05-2020

E-mail: npodoswa@yahoo.com.mx

DOI: 10.24875/GMM.20000241

Gac Med Mex. 2020;156:454-462

Disponible en PubMed

www.gacetamedicademexico.com

0016-3813/C 2020 Academia Nacional de Medicina de México, A.C. Publicado por Permanyer. Este es un artículo open access bajo la licencia CC BY-NC-ND (http://creativecommons.org/licenses/by-nc-nd/4.0/).
} 
En las últimas dos décadas y a raíz del esclarecimiento de varios de los mecanismos fisiopatológicos involucrados en el desarrollo de la enfermedad, han surgido diversos agentes terapéuticos llamados biológicos, los cuales se han convertido en pilares en el tratamiento de los pacientes con psoriasis. Para 2013, aproximadamente $25 \%$ de los pacientes con psoriasis moderada a severa ya eran tratados en el mundo con medicamentos biológicos. ${ }^{2,3}$

\section{Objetivos de las recomendaciones}

Con la finalidad de elaborar recomendaciones prácticas que permitan mantener al dermatólogo actualizado en el campo del manejo óptimo de los medicamentos biológicos, el grupo de expertos de PSOMEX (grupo mexicano para el estudio de psoriasis y enfermedades inmunomediadas) se reunió para elaborar dichas recomendaciones, las cuales están basadas en la mejor evidencia disponible y en la experiencia clínica de los integrantes del grupo.

\section{Método}

Se definieron múltiples preguntas relevantes concernientes al tratamiento biológico en psoriasis. Se realizó una investigación extensa de la literatura en PubMed/Medline para identificar reportes y series de casos, artículos de revisión y ensayos clínicos en los que se utilizaron los términos "psoriasis" y "biológicos". La revisión fue limitada a artículos publicados en inglés y español. Las respuestas se formularon conforme las evidencias de dicha revisión e incluyeron, además, la experiencia individual de los expertos. Conforme el método Delphi, se realizó una votación para lograr nivel el de acuerdo de las respuestas, definiéndose consenso cuando $70 \%$ de los participantes lograba acuerdo respecto a la recomendación.

\section{Recomendaciones}

\section{1. ¿Qué es un medicamento biológico y con cuáles} contamos?

El término "medicamento biológico" se refiere a anticuerpos monoclonales y proteínas de fusión diseñadas para bloquear de forma selectiva moléculas biológicas (citocinas, enzimas, etcétera).

En la actualidad, para el tratamiento de la psoriasis y artritis psoriática se dispone de agentes anti-TNF (infliximab, etanercept, adalimumab, certolizumab pegol y golimumab), agentes anti-IL12/anti-IL23
Tabla 1. Agentes biológicos para el tratamiento de la psoriasis

\begin{tabular}{l|l}
\hline Tipo de biológico & Medicamento \\
\hline Anti-TNF & $\begin{array}{l}\text { Infliximab, etanercept, adalimumab, } \\
\text { certolizumab pegol, golimumab }\end{array}$ \\
\hline Anti-IL12/anti-IL23 & Ustekinumab \\
\hline Anti-IL17 & Ixekizumab, secukinumab, brodalumab \\
\hline Anti-IL23 & Guselkumab, tildrakizumab y risankizumab
\end{tabular}

(ustekinumab), anti-IL17 (ixekizumab, secukinumab y brodalumab) y anti-IL23 (guselkumab, tildrakizumab y risankizumab) (Tabla 1).4-10
2. ¿Quién es candidato a un medicamento biológico?

Los candidatos a un medicamento biológico son los pacientes con psoriasis moderada a severa en quienes los tratamientos sistémicos convencionales han presentado falla terapéutica primaria o secundaria, estén contraindicados o no sean tolerados; ${ }^{11}$ así como los pacientes con psoriasis moderada a severa catalogada como de "alta necesidad", en la que el biológico puede ser indicado como de primera línea (Tabla 2).

3. ¿Quién indica el manejo biológico?

El tratamiento debe ser indicado y supervisado por dermatólogos con experiencia en el diagnóstico y tratamiento de la psoriasis y con amplio conocimiento en el manejo de agentes biológicos.

\section{4. ¿Cómo seleccionar el agente biológico?}

La decisión de cuál agente es el apropiado para iniciar debe ser individualizada, tomando en consideración diversos factores (ver evaluación pretratamiento)..$^{12,13}$

\section{Contraindicaciones absolutas}

- Hipersensibilidad al medicamento.

- Infecciones graves activas.

- Historia familiar o personal de enfermedad desmielinizante contraindica el uso de anti-TNF.

- Insuficiencia cardiaca grave grado III o IV de la clasificación de la New York Heart Association contraindica el uso de anti-TNF.

Con historia familiar o personal de enfermedad inflamatoria intestinal se sugiere que se evite el uso de agentes anti-IL17.

\section{Precauciones en la prescripción}

Anti-TNF debe prescribirse con cautela en pacientes con las siguientes condiciones: 
Tabla 2. Categorización de la gravedad de la psoriasis

\begin{tabular}{|c|c|c|c|c|}
\hline \multirow[t]{2}{*}{ Instrumento } & \multicolumn{4}{|c|}{ Gravedad } \\
\hline & Leve & Moderada & Severa & Alta necesidad \\
\hline PASI & $0-5$ & $5-10$ & $>10$ & $\begin{array}{l}\text { - Afectación de áreas de alto impacto: } \\
\text { cara, manos, pies y genitales. } \\
\text { - Presencia de artropatía psoriática. } \\
\text { - Generalización rápida. } \\
\text { - Resistencia a tratamientos. } \\
\text { - Formas graves, eritrodermia, } \\
\text { formas postulosas. }\end{array}$ \\
\hline BSA & $<3 \%$ & $3-8 \%$ & $>10 \%$ & \\
\hline DLQI & $2-5$ & $6-10$ & $11-20$ & $21-30$ \\
\hline PSSI & $\begin{array}{c}\text { Afectación < } 50 \% \text { de } \\
\text { la superficie, eritema, } \\
\text { infiltración y prurito } \\
\text { leves o mínimos }\end{array}$ & $\begin{array}{c}\text { Afectación }<50 \% \text { de } \\
\text { la superficie, eritema, } \\
\text { infiltración y prurito } \\
\text { moderados }\end{array}$ & $\begin{array}{c}\text { Afectación > } 50 \% \\
\text { de la superficie, eritema, } \\
\text { infiltración y prurito moderado o } \\
\text { grave, o presencia de alopecia } \\
\text { cicatrizal o afectación } \\
\text { en áreas visibles de la cara }\end{array}$ & \\
\hline
\end{tabular}

- Historia de infecciones recurrentes o infección grave previa.

- Historia de enfermedades hematológicas.

- Insuficiencia cardiaca grado I o II de la clasificación de la New York Heart Association.

- Historia personal o familiar de lupus.

- Potencial de embarazo y lactancia (ver apartado correspondiente).

\section{Evaluación pretratamiento}

Antes de iniciar el tratamiento con agentes biológicos se debe obtener una evaluación basal del paciente, para lo cual deberá realizarse historia clínica y examen físico completos, con especial atención en la forma clínica de la psoriasis, tratamientos previos, comorbilidades presentes (artritis psoriásica y tipo de artritis, historia de neoplasias, infecciones crónicas, linfadenopatías, enfermedad neurológica, cardiaca o autoinmune, etcétera), preferencias y disponibilidad para el almacenamiento y aplicación del medicamento.

El paciente debe ser ampliamente informado acerca de los riesgos y beneficios del tratamiento, así como de las precauciones que debe tomar durante el mismo. ${ }^{13,14}$

8. Exámenes de laboratorio y gabinete recomendados

Se recomienda la realización de los siguientes estudios a todos los pacientes antes del inicio del tratamiento biológico: ${ }^{14,15}$

- Biometría hemática completa, panel metabólico completo que incluya pruebas de función hepática, examen serológico para virus de la inmunodeficiencia humana, hepatitis $\mathrm{B}$ y $\mathrm{C}$, prueba de embarazo, prueba cutánea de derivado proteico purificado (para detección de tuberculosis), prueba de liberación de interferón gamma y telerradiografía de tórax.

- Panel inmunológico (anticuerpos antinucleares, anti-ADN), que, si bien no es estrictamente necesario, se recomienda si existen antecedentes familiares de lupus 0 en pacientes que durante el tratamiento desarrollen síntomas o signos sugestivos de lupus. La positividad por sí sola es inespecífica y no excluye que se inicie el tratamiento en un paciente.

\section{Vacunación}

Se debe tratar de actualizar el esquema de vacunación antes de iniciar el tratamiento biológico; durante este deben evitarse vacunas de virus vivos 0 vivos atenuados. La recomendación es no vacunar dos semanas antes de iniciar el tratamiento con agentes biológicos y esperar seis meses después de suspender el biológico para vacunar (12 meses para la vacuna de varicela zóster). Se puede reiniciar el tratamiento cuatro semanas después de la vacunación. Durante el tratamiento biológico se pueden aplicar vacunas de virus inactivados y se recomienda aplicar las vacunas de influenza y neumocócica durante el mismo. Tampoco deberán administrarse vacunas vivas o vivas atenuadas a recién nacidos que hayan sido expuestos a agentes biológicos in utero; deberá diferirse la vacunación por espacio de seis meses. ${ }^{16}$ 
10. Transición de tratamiento sistémico a biológico o de biológico a biológico

Si se presenta falla primaria o secundaria, la transición puede llevarse a cabo de forma directa, incluso con sobreposición con el tratamiento sistémico o biológico previo. Si se requiere la transición por el desarrollo de un evento adverso, se recomienda iniciar el agente biológico una vez que ha desaparecido el efecto secundario no deseado. ${ }^{17-19}$

\section{Monitoreo durante el tratamiento biológico y} evaluación de la respuesta

Durante el tratamiento, los pacientes deben ser evaluados periódicamente para vigilar la respuesta al tratamiento, así como el posible desarrollo de complicaciones. Los intervalos de revisión de los pacientes son variables, pero se recomienda como mínimo una evaluación trimensual.

Se recomienda la realización de los estudios de laboratorio a intervalos de tres a seis meses o antes, en caso de la exposición a riesgos o el desarrollo de signos o síntomas que sugieran efectos adversos..$^{20-22}$

12. La optimización del tratamiento biológico: incremento de dosis, la modificación en los intervalos de aplicación, las combinaciones

Ante una respuesta parcial o pérdida de la efectividad, antes de cambiar a otro agente biológico se recomienda siempre primero intentar optimizar el tratamiento con las siguientes estrategias:

- Incrementar la dosis del medicamento o acortar los intervalos de aplicación (Tabla 3).

- Combinar con agentes tópicos (esteroides tópicos, análogos de vitamina $D$, queratolíticos, reductores, retinoides), fototerapia o medicamentos sistémicos convencionales (metotrexato, ciclosporina por periodos cortos).

- Eliminar o modificar factores que contribuyan a la pérdida de efectividad: comorbilidades, infecciones intercurrentes, sobrepeso, tabaquismo, ingesta de alcohol y factores dietéticos. ${ }^{18,23-26}$

No se recomienda la combinación de dos o más agentes biológicos.

13. El intercambio de medicamentos (switching)

Se recomienda realizar intercambio del medicamento por falla terapéutica, ya sea primaria o secundaria, que no responda a la optimización o cuando se desarrolle un evento adverso relacionado con los medicamentos. Si se realiza por falla terapéutica, no es necesario esperar un tiempo para reiniciar con un biológico diferente, con el esquema habitual de dosificación (dosis de inducción y mantenimiento). Si el intercambio está en relación con el desarrollo de un evento adverso, se deberá esperar a que el evento se haya resuelto para reiniciar con otro biológico.

El intercambio puede ocurrir entre medicamentos del mismo grupo terapéutico si se requiere por falla terapéutica. Si el cambio es motivado por el desarrollo de eventos adversos considerados como de "clase", se aconseja cambiar a un medicamento con otro mecanismo de acción. ${ }^{27}$

14. Tratamiento intermitente versus tratamiento continuo

En general, con los agentes biológicos no se recomiendan los tratamientos intermitentes o rotacionales.

15. Cuándo suspender el biológico

- Falla primaria: incapacidad para lograr por lo menos una reducción de $50 \%$ en el Psoriasis Area and Severity Index (PASI 50) en los lapsos establecidos para cada medicamento.

- Falla secundaria que no responde a estrategias de optimización.

- Desarrollo de evento adverso grave.

- Cirugías mayores electivas.

- Necesidad de vacunación.

- Embarazo (ver apartado correspondiente).

16. Reinicio del tratamiento posterior a la suspensión

Etanercept, adalimumab, ustekinumab, ixekizumab, secukinumab se pueden reiniciar con buena recaptura de la respuesta.

El reinicio con dosis de inducción depende de la severidad del padecimiento y del lapso transcurrido desde la última dosis. Será necesario considerarlo si han pasado más de tres o cuatro vidas media del medicamento desde la última administración.

No se aconseja reiniciar infliximab después de suspensiones prolongadas (más de 12 semanas), debido al alto riesgo de pérdida de efectividad, así como de reacciones adversas mediadas por anticuerpos antimedicamento. ${ }^{28-30}$

\section{Interacciones medicamentosas}

Los medicamentos biológicos pueden acelerar el metabolismo de múltiples medicamentos o incrementar su respuesta, por lo cual se debe vigilar las interacciones medicamentosas (Tabla 4).

\section{Los agentes biológicos en poblaciones y situaciones especiales}

\section{En edad pediátrica}

En niños candidatos a tratamiento biológico se puede utilizar adalimumab a partir de los cuatro años 

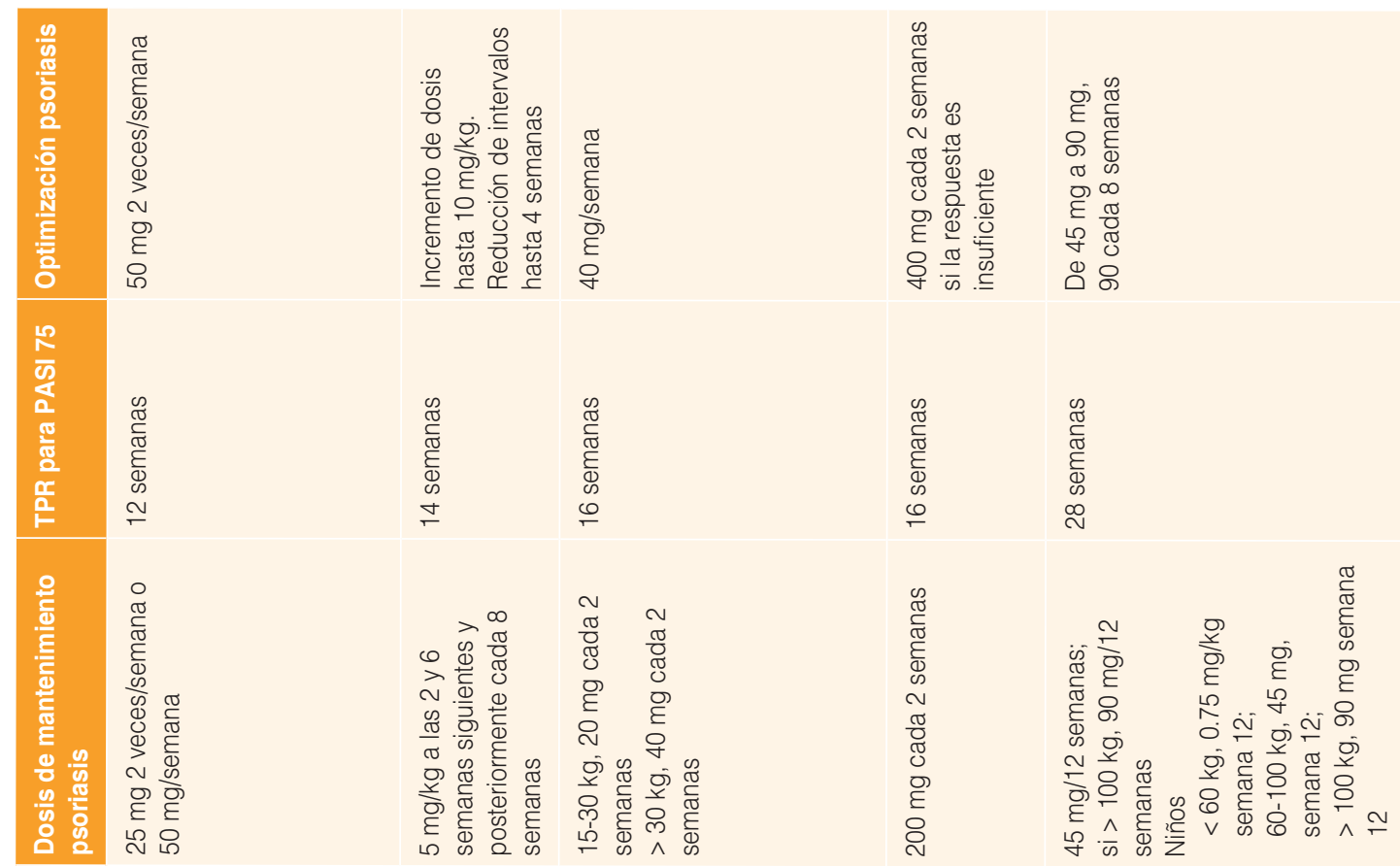

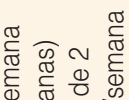

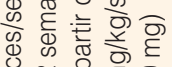

过 $\simeq \therefore$

$\sim \circ$

을

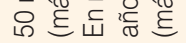

$\frac{{ }^{2}}{\sum^{2}}$

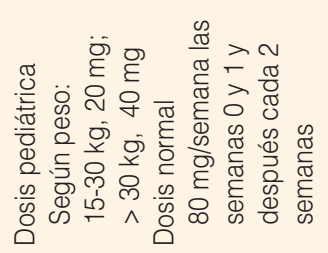

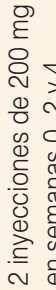

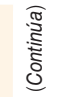
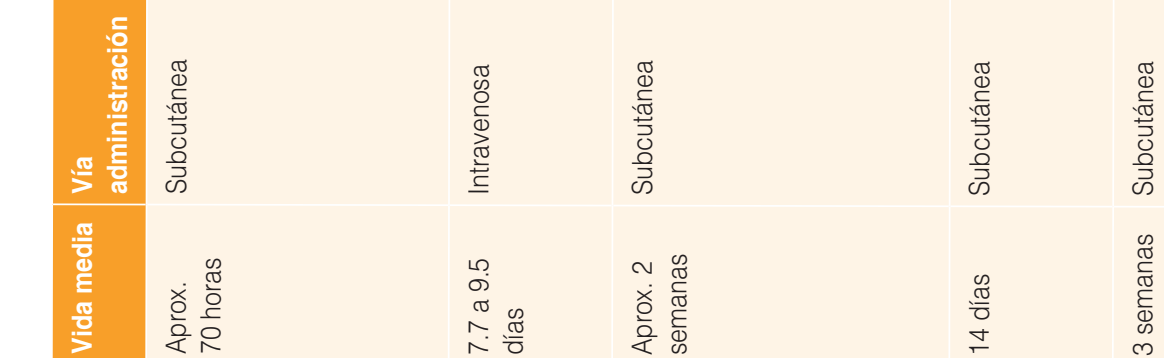

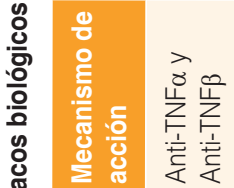
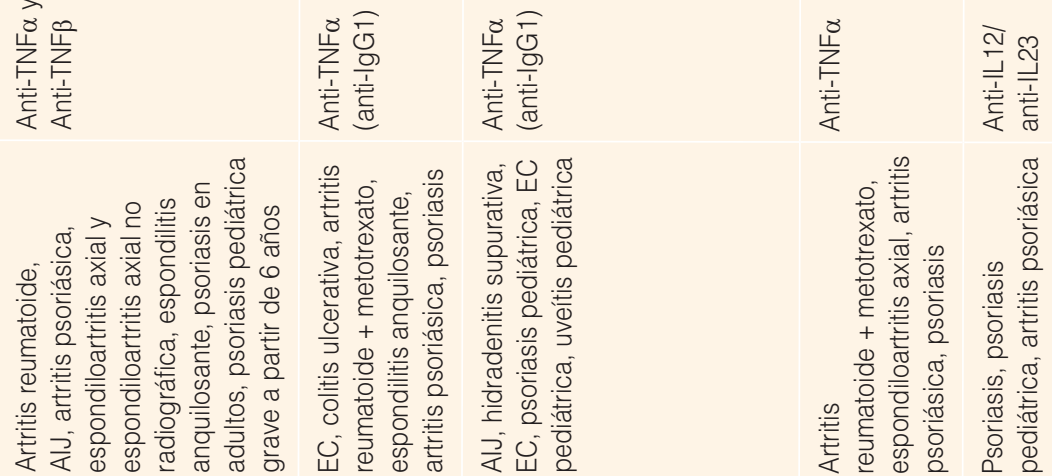

兽

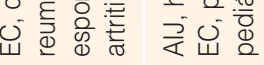

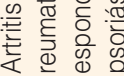
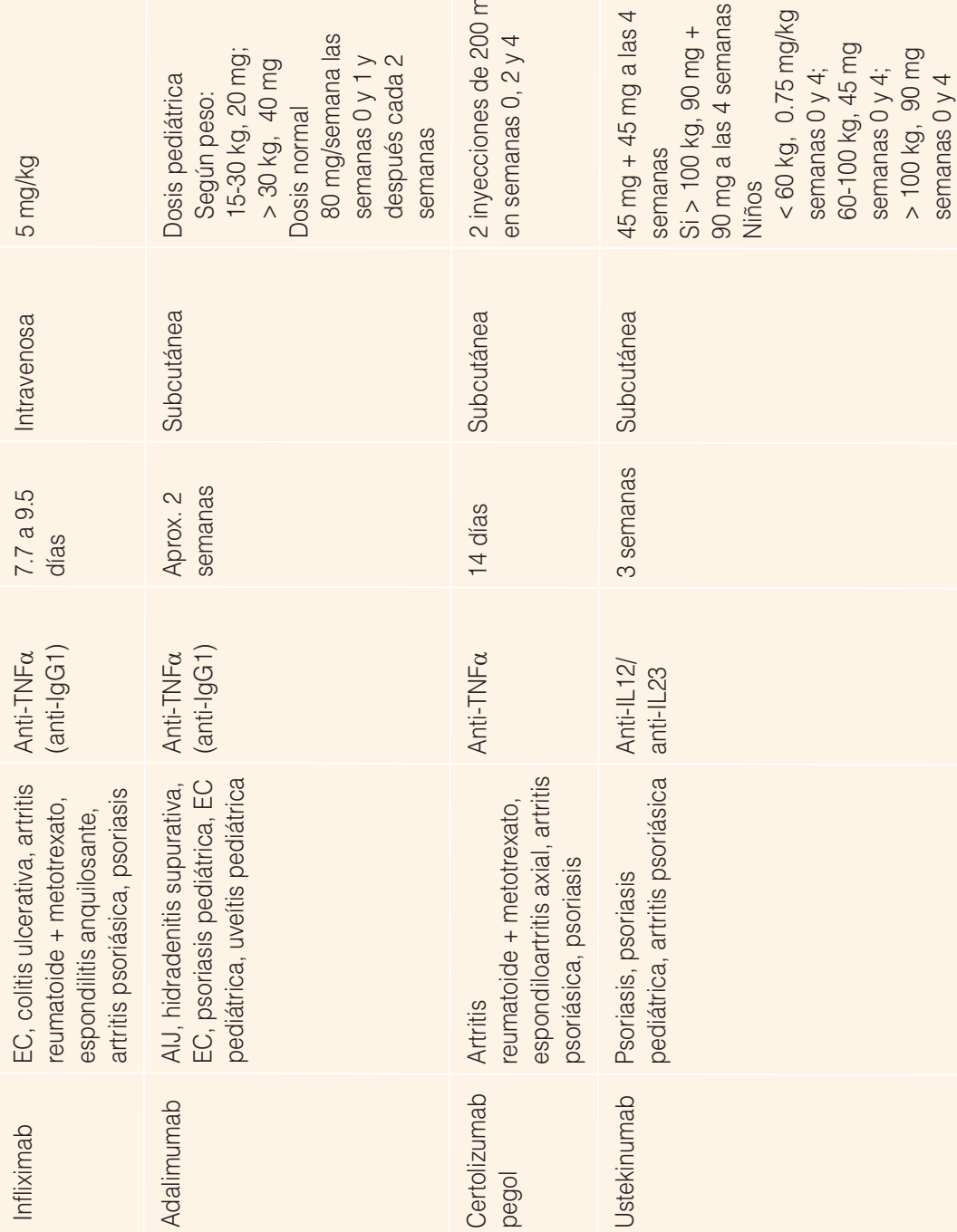


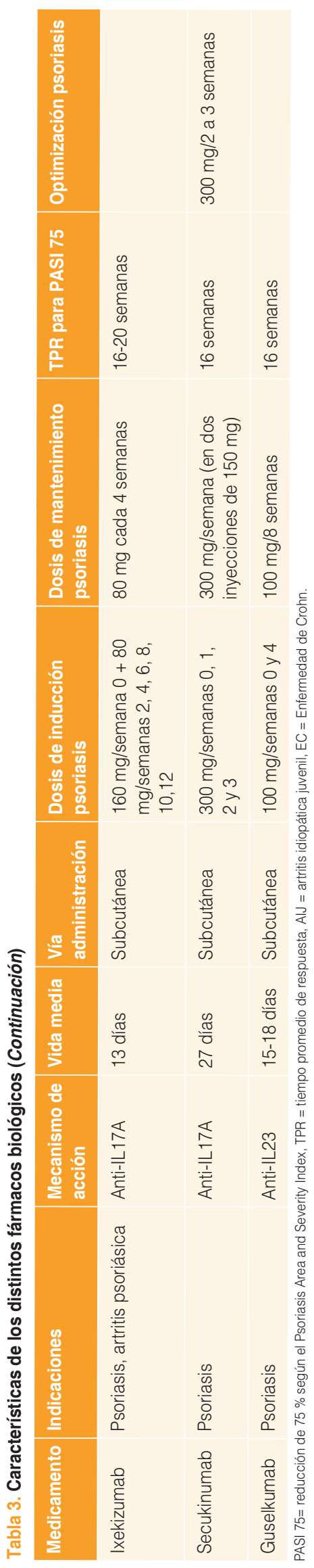

de edad, etanercept a partir de los seis años y ustekinumab a partir de los 12 años. ${ }^{31-34}$

2. En población geriátrica (> 65 años)

El tratamiento biológico es eficaz y seguro en esta población. Pudiera haber un incremento en el riesgo de efectos adversos como infección, neoplasia o interacciones medicamentosas. ${ }^{35}$

3. Embarazo, lactancia y potencial reproductivo

Se recomienda evitar el embarazo durante el tratamiento biológico.

No se conocen interacciones entre tratamientos biológicos y métodos anticonceptivos.

Con planes de embarazo se recomienda suspender los agentes biológicos durante los siguientes lapsos: etanercept, tres semanas; infliximab, seis semanas; adalimumab, seis meses; ustekinumab, 15 semanas. $^{36}$

De necesitar tratamiento biológico durante el embarazo, los agentes anti-TNF serían los de primera elección y de estos, certolizumab pegol en primera línea seguido de etanercept. ${ }^{37}$

Se recomienda suspender los anticuerpos monoclonales en mujeres embarazadas a las 16 semanas de gestación, para evitar el paso transplacentario de anticuerpos.

Es necesario informar a la paciente embarazada en tratamiento biológico de los riesgos "teóricos" de abortos espontáneos y de inmunosupresión en el recién nacido.

Se considera que la lactancia es segura durante el tratamiento con anti-TNF, perfil de seguridad que puede aplicarse a los agentes anti-IL17 y anti-IL23. ${ }^{38}$

Los pacientes masculinos deberán ser informados sobre la capacidad de los agentes anti-TNF para mejorar la capacidad de fertilización.

No se han reportado alteraciones en la concepción ni en el desarrollo del producto de pacientes masculinos bajo tratamiento con anti-TNF. ${ }^{39,40}$

\section{Psoriasis eritrodérmica}

Infliximab es el medicamento biológico de elección para tratar la psoriasis eritrodérmica rápidamente progresiva e inestable. No se recomienda utilizar este medicamento como medicamento de rescate. ${ }^{41}$

\section{Artritis psoriática}

Los agentes anti-TNF se recomiendan como agentes de primera línea en el tratamiento de pacientes con artritis psoriática grave, con o sin afectación grave de la piel. Como segunda línea de tratamiento se recomiendan los agentes anti-IL17 seguidos de ustekinumab. El tratamiento debe llevarse en interconsulta con el reumatólogo. ${ }^{42}$ 
Tabla 4. Algunas de las principales interacciones mayores y menores de los agente biológicos para tratar la psoriasis

\begin{tabular}{|c|c|c|}
\hline Medicamento & $\begin{array}{l}\text { Interacciones mayores } \\
\text { (no se recomienda administrar) }\end{array}$ & $\begin{array}{l}\text { Interacciones menores } \\
\text { (se recomienda vigilar-puede afectar) }\end{array}$ \\
\hline Anti-TNF & $\begin{array}{l}\text { - Vacunas de virus vivos } \\
\text { - Otro biológico }\end{array}$ & $\begin{array}{l}\text { - Insulina y antidiabéticos orales (riesgo de hipoglucemia) } \\
\text { - Antihipertensivos (amlodipino, nimodipino) } \\
\text { - Estatinas (atorvastatina, simvastatina) } \\
\text { - Benzodiacepinas (diazepam, alprazolam) } \\
\text { - Anticoagulantes (warfarina) }\end{array}$ \\
\hline Anti-IL12/anti-IL23 & $\begin{array}{l}\text { - Vacunas de virus vivos } \\
\text { - Otro biológico }\end{array}$ & $\begin{array}{l}\text { - Metilprednisolona } \\
\text { - Benzodiacepinas (diazepam) } \\
\text { - Anticonceptivos orales (etinil estradiol, etonogestrel) } \\
\text { - Estatinas (lovastatina, atorvastatina) } \\
\text { - Antihipertensivos (nicardipino, nifedipino) } \\
\text { - Anticoagulantes (warfarina) }\end{array}$ \\
\hline Anti-IL17 & $\begin{array}{l}\text { - Vacunas de virus vivos } \\
\text { - Otro biológico }\end{array}$ & $\begin{array}{l}\text { - Antiarrítmicos (amiodarona) } \\
\text { - Antihipertensivos (amlodipino, nicardipino) } \\
\text { - Benzodiacepina (clonazepam, diazepam) } \\
\text { - Anticonceptivos orales (drosperinona, norgestrel) } \\
\text { - Estatinas (simvastatina) } \\
\text { - AntiHER2 (everolimus) } \\
\text { - Anticoagulantes (warfarina) }\end{array}$ \\
\hline Anti-IL23 & $\begin{array}{l}\text { - Vacunas de virus vivos } \\
\text { - Otro biológico }\end{array}$ & $\begin{array}{l}\text { - Antiarrítmicos (amiodarona) } \\
\text { - Antihipertensivos (amlodipino, nicardipino) } \\
\text { - Anticoagulantes (warfarina) } \\
\text { - Analgésicos opiáceos (metadona) } \\
\text { - Benzodiacepinas (clonazepam, diazepam) } \\
\text { - Anticonceptivos orales (etinilestradiol, etonogestrel) }\end{array}$ \\
\hline
\end{tabular}

\section{Enfermedades virales crónicas}

- Virus de la inmunodeficiencia humana (VIH). Se pueden utilizar agentes biológicos en pacientes VIH positivos con psoriasis moderada a severa resistente a tratamientos sistémicos convencionales, en combinación con el tratamiento antirretroviral (HAART). Se sugiere utilizar etanercept como primera elección, si bien todos los anti-TNF, ustekinumab, secukinumab e ixekizumab han sido utilizados con buen perfil de seguridad y eficacia. Se recomienda el tratamiento en conjunción con infectología, así como el monitoreo regular de la carga viral y de posibles efectos adversos. ${ }^{43}$

- Hepatitis B y C. Antes de iniciar el manejo con un medicamento biológico siempre deberá interconsultarse con el especialista. Se recomienda ofrecer manejo antiviral antes de comenzar el tratamiento y durante este se deben monitorear las pruebas de función hepática, así como la carga viral.

Los agentes anti-TNF deben evitarse en pacientes con infección por el virus de la hepatitis $B$, porque se han asociado a reactivación de la infección; ustekinumab sería el agente de elección, con bajo riesgo de reactivación viral, seguido de agentes anti-IL17 y anti-IL23.

Los datos de los estudios sugieren que los anti-TNF pueden ser utilizados en pacientes con infección por virus de la hepatitis C; al respecto, la información acerca del uso de ustekinumab es escasa y contradictoria, por lo que se sugiere cautela. Aun no hay datos suficientes en cuanto a la utilización de agentes anti-IL17 o anti-IL23 en este contexto. ${ }^{44-49}$

\section{Tuberculosis latente}

Se recomienda iniciar con ustekinumab o agentes anti-IL17 en pacientes con tuberculosis latente que hayan completado por lo menos un mes de tratamiento antifímico. Etanercept es el agente anti-TNF que presenta el menor riesgo de reactivación. ${ }^{50,51}$

8. Enfermedad hepática: esteatohepatitis, cirrosis

Los agentes anti-TNF y ustekinumab pueden ser utilizados con cautela y en conjunto con el especialista. Los datos hasta el momento sugieren que los agentes anti-IL17 podrían también ser empleados con buen perfil de seguridad. ${ }^{22,53}$

\section{Trasplante de órganos}

En general, se recomienda utilizar agentes biológicos como tercera línea en pacientes con trasplante. 
La mayor parte de estos pacientes han sido tratados con etanercept, aunque ya existen algunos reportes de buena respuesta con la utilización de ixekizumab, con buen perfil de seguridad. ${ }^{54}$

\section{Agentes biológicos en cirugía}

En procedimientos quirúrgicos de bajo riesgo se considera que los agentes anti-TNF y ustekinumab no requieren ser suspendidos debido a infección posquirúrgica. En aquellos que presenten riesgos moderados 0 altos de complicaciones (infección 0 impacto negativo en cicatrización), la decisión de continuar o no con el tratamiento biológico debe ser individualizada tomando en cuenta el tipo de procedimiento, las comorbilidades del paciente, la severidad de la enfermedad y el riesgo de exacerbación tras la suspensión del biológico, así como la medicación concomitante.

De requerir la suspensión del agente biológico antes de la cirugía, se recomienda hacerlo con una anticipación de tres a cinco vidas media: en etanercept, dos a tres semanas; en adalimumab, seis a ocho semanas; en infliximab, cuatro a seis semanas; en ustekinumab, 12 semanas.

No hay datos suficientes para realizar recomendaciones específicas respecto a los agentes más nuevos, anti-IL17 e anti-IL23. ${ }^{55}$

\section{Agentes biológicos en hemodiálisis}

Se recomienda la utilización de agentes biológicos como primera línea en pacientes con enfermedad renal y en aquellos en hemodiálisis (anti-IL17, ustekinumab $\mathrm{y}$ anti-TNF). ${ }^{56}$

\section{Agentes biológicos en pacientes con cáncer}

En general, los agentes biológicos, incluyendo anti-TNF, ustekinumab y agentes anti-IL17 no están contraindicados en pacientes con historia personal de neoplasias sólidas, hematológicas o cutáneas no melanoma y melanoma. La decisión de iniciar tramiento con algún medicamente biológico en un paciente debe ser individualizada y siempre en conjunto con el oncólogo. ${ }^{57,58}$

\section{Sobrepeso y obesidad}

Se ha demostrado el efecto negativo del sobrepeso en el tratamiento biológico, con excepción de infliximab, el único medicamento dosificado por kilogramo de peso corporal, por lo que este es el de elección en pacientes con obesidad mórbida.

Podrá considerarse el incremento de la dosis o el acortamiento de los intervalos de aplicación en los pacientes con obesidad que no responden a las dosis estándar (ver optimización). ${ }^{59}$

\section{Conflicto de intereses}

La doctora Podoswa ha colaborado con los laboratorios Elly Lilly, Abbvie, Pfizer, Novartis y Janssen.

\section{Financiamiento}

La reunión para desarrollar el artículo de recomendaciones se llevó a cabo con el apoyo del laboratorios Eli Lilly y Compañía de México S. A de C. V.

\section{Responsabilidades éticas}

Protección de personas y animales. Los autores declaran que para esta investigación no se realizaron experimentos en seres humanos ni en animales.

Confidencialidad de los datos. Los autores declaran que en este artículo no aparecen datos de pacientes.

Derecho a la privacidad y consentimiento informado. Los autores declaran que en este artículo no aparecen datos de pacientes.

\section{Bibliografía}

1. Gelfand JM, Troxel AB, Lewis JD, Kurd SK, Shin DB, Wang X, et al. The risk of mortality in patients with psoriasis: results from a population-based study. Arch Dermatol. 2007;143:1493-1499.

2. Parisi R, Symmons DP, Griffiths CE, Ashcroft DM, Identification and Management of Psoriasis and Associated ComorbidiTy (IMPACT) project team. Global epidemiology of psoriasis: a systematic review of incidence and prevalence. J Invest Dermatol. 2013:133:377-385.

3. Yeung H, Takeshita J, Mehta NN, Kimmel SE, Ogdie A, Margolis DJ, etal. Psoriasis severity and the prevalence of major medical comorbidity: a population-based study. JAMA Dermatol. 2013:149:1173-1179.

4. Kerensky TA, Gottlieb AB, Yaniv S, Au SC. Etanercept: efficacy and safety for approved indications. Expert Opin Drug Saf. 2012;11:121-139.

5. Burmester GR, Panaccione R, Gordon, Mcllraith MJ, Lacerda APM. Adalimumab: long-term safety in 23,458 patients from global clinical trials in rheumatoid arthritis, juvenile idiopathic arthritis, ankylosing spondylitis, psoriatic arthritis, psoriasis and Crohn's disease. Ann Rheum Dis. 2013;72:517-524.

6. Chaudhari U, Romano P, Mulcahy LD, Dooley LT, Baker DG, Gottlieb AB. Efficacy and safety of infliximab monotherapy for plaque-type psoriasis: a randomised trial. Lancet. 2001;357(9271):1842-1847.

7. Gottlieb AB, Cooper KD, McCormick TS, Toichi E, Everitt DE, Frederick $B$, et al. A phase 1, double-blind, placebo-controlled study evaluating single subcutaneous administrations of a human interleukin-12/23 monoclonal antibody in subjects with plaque psoriasis. Curr Med Res Opin. 2007;23:1081-1092.

8. Leonardi C, Matheson R, Zachariae C, Cameron G, Li L, Edson-Heredia E, et al. Anti-interleukin-17 monoclonal antibody ixekizumab in chronic plaque psoriasis. N Engl J Med. 2012;366:1190-1199.

9. Gottlieb AB, Blauvelt A, Thaçi D, Leonardi CL, Poulin Y, Drew J, et al. Certolizumab pegol for the treatment of chronic plaque psoriasis: results through 48 weeks from 2 phase 3 , multicenter, randomized, double-blinded, placebo-controlled studies (CIMPASI-1 and CIMPASI-2). J Am Acad Dermatol. 2018;79:302-314.e6.

10. Reich K, Ortonne JP, Gottlieb AB, Terpstra IJ, Coteur G, Tasset C, et al. Successful treatment of moderate to severe plaque psoriasis with the PEGylated Fab' certolizumab pegol: results of a phase II randomized, placebo-controlled trial with a re-treatment extension. $\mathrm{Br} \mathrm{J}$ Dermatol. 2012;167:180-190.

11. Tan J, Bhambri S, Zeichner J. A practical approach to screening psoriasis patients for therapy with biologic agents. J Clin Aesthet Dermatol. 2008:1:50-54.

12. Kaushik SB, Lebwohl MG. Psoriasis: which therapy for which patient: psoriasis comorbidities and preferred systemic agents. J Am Acad Dermatol. 2019;80:27-40 
13. Menter A, Gottlieb A, Feldman SR, van Voorhees AS, Leonardi CL Gordon KB, et al. Guidelines of care for the management of psoriasis and psoriatic arthritis: section 1. Overview of psoriasis and guidelines of care for the treatment of psoriasis with biologics. J Am Acad Dermatol. 2008:58:826-850.

14. Halverstam CP, Lebwohl M. Practical management and monitoring of psoriasis in patients on biologic therapy. Psoriasis Forum. 2007;13:4-12.

15. Huang W, Cordoro KM, Taylor SL, Feldman SR. To test or not to test? An evidence-based assessment of the value of screening and monitoring tests when using systemic biologic agents to treat psoriasis. J Am Acad Dermatol. 2008;58:970-977

16. Lebwohl M, Bagel J, Gelfand JM, Gladman D, Gordon KB, Hsu S, et al. From the Medical Board of the National Psoriasis Foundation: monitoring and vaccinations in patients treated with biologics for psoriasis. J Am Acad Dermatol. 2008:58:94-105.

17. Ganzetti G, Campanati A, Bernardini ML, Bettacchi A, Brandozzi G Brisigotti $\mathrm{V}$, et al. The transitioning from conventional therapy to biological treatment in psoriatic patients: STRATOS, a project of Marche Region. G Ital Dermatol Venereol. 2016:151:340-346.

18. Mrowietz U, de Jong EM, Kragballe K, Langley R, Nast A, Puig L, et al. A consensus report on appropriate treatment optimization and transitioning in the management of moderate-to-severe plaque psoriasis. $\mathrm{J}$ Eur Acad Dermatol Venereol. 2014;28:438-453.

19. Ganzetti G, Campanati A, Bettacchi A, Brandozzi G, Brisigotti V, Bugatti $L$, et al. Switching from a biological therapy to another biologic agent in psoriatic patients: the experience of PsOMarche group. G Ital Dermatol Venereol. 2018;153:5-10.

20. Armstrong AW, Siegel MP, Bagel J, Boh EE, Buell M, Cooper KD, et al From the Medical Board of the National Psoriasis Foundation: treatment targets for plaque psoriasis. J Am Acad Dermatol. 2017;76:290-298.

21. Menter A, Strober BE, Kaplan DH, Kivelevitch D, Prater EF, Stoff B, et al. Joint AAD-NPF guidelines of care for the management and treatment of psoriasis with biologics. J Am Acad Dermatol. 2019;80:1029-1072.

22. Smith CH, Jabbar-Lopez ZK, Yiu ZZ, Bale T, Burden AD, Coates LC et al. British Association of Dermatologists guidelines for biologic therapy for psoriasis 2017. Br J Dermatol. 2017:177:628-636.

23. Castaldo G, Galdo G, Rotondi-Aufiero F, Cereda E. Very low-calorie ketogenic diet may allow restoring response to systemic therapy in relapsing plaque psoriasis. Obes Res Clin Pract. 2016;10:348-352.

24. Barrea L, Balato N, Di Somma C, Macchia PE, Napolitano M, Savanelli MC, et al. Nutrition and psoriasis: is there any association between the severity of the disease and adherence to the Mediterranean diet? J Transl Med. 2015:13:18

25. Millsop JW, Bhatia BK, Debbaneh M, Koo J, Liao W. Diet and psoriasis, part III: role of nutritional supplements. J Am Acad Dermatol. 2014;71:561-569.

26. Armstrong AW, Bagel J, van Voorhees AS, Robertson AD, Yamauchi PS. Combining biologic therapies with other systemic treatments in psoriasis: evidence-based, best-practice recommendations from the Medical Board of the National Psoriasis Foundation. JAMA Dermatol. 2015;151: 432-438.

27. Piaserico S, Cazzaniga S, Chimenti S, Giannetti A, Maccarone M, Picardo $\mathrm{M}$, et al. Efficacy of switching between tumor necrosis factor-alfa inhibitors in psoriasis: results from the Italian Psocare registry. J Am Acad Dermatol. 2014;70:257-262

28. Gordon KB, Gottlieb AB, Leonardi CL, Elewski BE, Wang A, Jahreis A, et al. Clinical response in psoriasis patients discontinued from and then reinitiated on etanercept therapy. J Dermatolog Treat. 2006:17:9-17.

29. Daudén E, Griffiths CE, Ortonne JP, Kragballe K, Molta CT, Robertson D, et al. Improvements in patient-reported outcomes in moderate-to-severe psoriasis patients receiving continuous or paused etanercept treatment over 54 weeks: the CRYSTEL study. J Eur Acad Dermatol Venereol. 2009;23:1374-1382.

30. Gordon KB, Gottlieb AB, Langely RG, van de Kerkhof P, Belasco KT, Sundaram $\mathrm{M}$, et al. Adalimumab retreatment successfully restores clinical response and health-related quality of life in patients with moderate to severe psoriasis who undergo therapy interruption. J Eur Acad Dermatol Venereol. 2015;29:767-776.

31. Landells I, Paller AS, Pariser D, Kricorian G, Foehl J, Molta C, et al. Efficacy and safety of etanercept in children and adolescents aged $>$ or $=8$ years with severe plaque psoriasis. Eur $\mathrm{J}$ Dermatol. 2010:20:323-328

32. Langley RG, Paller AS, Hebert AA, Creamer K, Weng HH, Jahreis A, et al. Patient-reported outcomes in pediatric patients with psoriasis undergoing etanercept treatment: 12-week results from a phase III randomized controlled trial. J Am Acad Dermatol. 2011;64:64-70.

33. Sanclemente G, Murphy R, Contreras J, García H, Bonfill-Cosp X. Anti-TNF agents for paediatric psoriasis. Cochrane Database Syst Rev. 2015;2015:CD010017.

34. Klufas DM, Wald JM, Strober BE. Treatment of moderate to severe pediatric psoriasis: a retrospective case series. Pediatr Dermatol. 2016;33:142-149.
35. Ricceri F, Bardazzi F, Chiricozzi A, Dapavo P, Ferrara F, Mugheddu C, et al. Elderly psoriatic patients under biological therapies: an Italian experience. J Eur Acad Dermatol Venereol. 2019;33:143-146.

36. Schaufelberger BW, Horn E, Cather JC, Rahawi KW. Pregnancy outcomes in women exposed to ustekinumab in the psoriasis clinical development program. J Am Acad Dermatol. 2014;70:AB178.

37. Mariette X, Förger F, Abraham B, Flynn AD, Moltó A, Flipo RM, et al. ack of placental transfer of certolizumab pegol during pregnancy: results from CRIB, a prospective, postmarketing, pharmacokinetic study. Ann Rheum Dis. 2018;77:228-233.

38. Clowse ME, Förger F, Hwang C, Thorp J, Dolhain RJ, van Tubergen A, et al. Minimal to no transfer of certolizumab pegol into breast milk: results from CRADLE, a prospective, postmarketing, multicentre, pharmacokinetic study. Ann Rheum Dis. 2017;76:1890-1896.

39. Flood KS, Porter ML, Kimball AB. Use of biologics in pregnancy: limitations stemming from clinical trials and registry experience. J Eur Acad Dermatol Venereol. 2019;33:e276-e277.

40. Porter ML, Lockwood SJ, Kimball AB. Update on biologic safety for patients with psoriasis during pregnancy. Int $\mathrm{J}$ Womens Dermatol. 2017;3:21-25

41. Rosenbach M, Hsu S, Korman NJ, Lebwohl MG, Young M, Bebo BF Jr, et al. Treatment of erythrodermic psoriasis: from the medical board of the National Psoriasis Foundation. J Am Acad Dermatol. 2010;62:655-662.

42. Smolen JS, Landewé R, Bijlsma J, Burmester G, Chatzidionysiou K, Dougados $M$, et al. EULAR recommendations for the management of rheumatoid arthritis with synthetic and biological disease-modifying antirheumatic drugs: 2016 update. Ann Rheum Dis. 2017;76:960-977.

43. Nakamura M, Abrouk M, Farahnik B, Zhu TH, Bhutani T. Psoriasis treatment in HIV-positive patients: a systematic review of systemic immunosuppressive therapies. Cutis. 2018;101:38;42-56.

44. Brunasso AM, Puntoni M, Gulia A, Massone C. Safety of anti-tumour necrosis factor agents in patients with chronic hepatitis $\mathrm{C}$ infection: a systematic review. Rheumatology (Oxford). 2011:50:1700-1711.

45. Di Lernia V. Treatment of psoriasis in patients with hepatitis $C$ virus infection. Clin Exp Dermatol. 2011;36:417.

46. Navarro R, Vilarrasa E, Herranz P, Puig L, Bordas X, Carrascosa JM, et al. Safety and effectiveness of ustekinumab and antitumour necrosis factor therapy in patients with psoriasis and chronic viral hepatitis B or $\mathrm{C}$ : a retrospective, multicentre study in a clinical setting. $\mathrm{Br} \mathrm{J}$ Dermatol. 2013:168:609-616.

47. Rokhsar C, Rabhan N, Cohen SR. Etanercept monotherapy for a patient with psoriasis, psoriatic arthritis, and concomitant hepatitis C infection. J Am Acad Dermatol. 2006:54:361-362.

48. Aslanidis S, Vassiliadis T, Pyrpasopoulou A, Douloumpakas I, Zamboulis C. Inhibition of TNFalpha does not induce viral reactivation in patients with chronic hepatitis C infection: two cases. Clin Rheumatol. 2007;26:261-264.

49. Salvi M, Macaluso L, Luci C, Mattozzi C, Paolino G, Aprea Y, et al. Safety and efficacy of anti-tumor necrosis factors $\alpha$ in patients with psoriasis and chronic hepatitis C. World J Clin Cases. 2016;4:49-55.

50. Dobler CC. Biologic agents and tuberculosis. Microbiol Spectr. 2016;4.

51. Shobha V, Chandrashekara S, Rao V, Desai A, Jois R, Dharmanand BG, et al. Biologics and risk of tuberculosis in autoimmune rheumatic diseases: a real-world clinical experience from India. Int J Rheum Dis. 2019;22:280-287.

52. Vilarrasa E, Puig L. Psoriasis: biologic treatment and liver disease. World J Dermatol. 2014;3:76-85.

53. Campanati A, Ganzetti G, Di Sario A, Damiani A, Sandroni L, Rosa L, et al. The effect of etanercept on hepatic fibrosis risk in patients with non-alcoholic fatty liver disease, metabolic syndrome, and psoriasis. J Gastroenterol. 2013:48:839-846.

54. Prussick R, Wu JJ, Armstrong AW, Siegel MP, Van Voorhees AS. Psoriasis in solid organ transplant patients: best practice recommendations from The Medical Board of the National Psoriasis Foundation. J Dermatolog Treat. 2018;29:329-333

55. Galiano-Mejías S, Carretero G, Ferrandiz C, Vanaclocha F, Daudén E, Gómez-García FJ, et al. Manejo de los tratamientos biológicos en pacientes con psoriasis moderada-grave sometidos a intervenciones quirúrgicas en el registro español Biobadaderm. Actas Dermosifiliogr. 2017; $108: 52-58$

56. Larquey M, Girard C, Sbidian E, Richard MA, Aubin F, Schmutz JL. Efficacy of biologics in psoriasis patients under hemodialysis. Eur $\mathrm{J}$ Dermatol. 2017;27:531-533.

57. Peleva E, Exton LS, Kelley K, Kleyn CE, Mason KJ, Smith CH. Risk of cancer in patients with psoriasis on biological therapies: a systematic review. Br J Dermatol. 2018;178:103-113.

58. Chen Y, Friedman M, Liu G, Deodhar A, Chu CQ. Do tumor necrosis factor inhibitors increase cancer risk in patients with chronic immune-mediated inflammatory disorders? Cytokine. 2018;101:78-88

59. Puig L. Obesity and psoriasis: body weight and body mass index influence the response to biological treatment. J Eur Acad Dermatol Venereol. 2011;25:1007-1011. 